\title{
UMA PAISAGEM DA EPISTEMOLOGIA E Metodologia em CoMunicaÇão
}

\author{
Sandra Marinho \& Miguel Vicente Mariño
}

Aolongo doséculoXX, a investigação em Comunicação consolidou progressivamente a sua presença e relevância no seio das Ciências Sociais. As transformações tecnológicas e sociais, contínuas e aceleradas, vivenciadas desde a chegada da sociedade em rede (Castells, 2000) estabeleceram a Comunicação como uma das principais áreas emergentes da investigação científica em todo o mundo. Enraizada num território interdisciplinar e combinando várias Ciências Sociais - como a Sociologia, a Psicologia ou a Ciência Política - com as Humanidades, a Comunicação tem provado ser um domínio de investigação indispensável e um novo e atrativo campo profissional e académico. Desde os seus passos iniciais, ainda hesitantes, - ao explorar os efeitos sociais da comunicação de massa - até uma investigação teoricamente robusta e empiricamente orientada, a investigação em Comunicação tem percorrido um longo caminho e enfrenta agora novos desafios teóricos e práticos, para que possa servir como uma referência basilar e sólida para compreender as sociedades contemporâneas (Norderstreng, 2004).

Ao longo dos anos 9o, houve uma expansão dos cursos de Média e Comunicação oferecidos por instituições de ensino superior em todo o mundo, uma tendência ainda visível no século XXI, o que comprova a procura social e profissional por especialização neste campo. O número de estudantes não tem diminuído e esta procura estável - se não crescente - tem impulsionado uma evolução paralela em todas as atividades de investigação relacionadas com a área. $O$ desenvolvimento de competências profissionais e intelectuais exigiu uma evolução em sincronia, no que toca à consolidação do estatuto científico desta disciplina, em termos nacionais e internacionais. Reconhecimento institucional e visibilidade têm sido as duas faces de uma luta contínua nesta área emergente, que pretende atingir um estatuto semelhante ao de disciplinas próximas e, assim, permitir que a investigação em Comunicação interaja e dialogue com essas áreas de uma forma equilibrada, baseada em reconhecimento mútuo e cooperação intelectual. $A$ luta científica por recursos que são escassos, tais como financiamento, presença institucional e reconhecimento público, ainda é uma realidade e tem servido de pano de fundo para os esforços da Investigação em Comunicação no sentido de ser percebida como uma área autónoma e específica da produção de conhecimento científico (Craig, 2008).

Para atingir este estatuto e este prestígio social, a investigação em Comunicação não pode abster-se de uma discussão aberta acerca dos seus fundamentos epistemológicos e metodológicos (Craig, 1999). O facto de lidar com um cenário de evolução tecnológica acelerada como um dos seus principais tópicos de estudo não deverá implicar resultados de investigação pouco fundamentados, se se pretende que esta estrutura 
científica se torne num ponto de vista sólido para compreender as transformações sociais da atualidade.

Este número da Comunicação e Sociedade é sustentado por este posicionamento intelectual e reúne um conjunto relevante de artigos que exploram diversos aspetos centrais indispensáveis para se conduzir estudos bem fundamentados. Aprofundarmos a nossa relação, como académicos da área dos Média e da Comunicação, com a forma como planeamos e aplicamos a investigação que desenvolvemos é essencial para fortalecer a posição institucional do nosso campo. As condições sociais de produção de conhecimento tornaram-se num objeto de pesquisa atrativo, reivindicando a necessidade de reflexão epistemológica sobre a investigação com um enfoque mais amplo nos fenómenos sociais: metas de curto prazo devem ser complementadas com uma análise mais aprofundada sobre o que, por que e como selecionamos e abordamos tópicos, questões e hipóteses de investigação. Pesquisar num domínio tecnologicamente avançado não pode implicar deixar para trás algumas das principais e mais amplas questões filosóficas sobre o nosso papel como cientistas sociais e intelectuais. Consequentemente, continua a ser necessário reclamar a necessidade de abordagens relevantes do ponto de vista da Epistemologia e das Metodologias, como uma forma de nos abstrairmos das rotinas das nossas práticas científicas e das exigências institucionais, que afetam as vidas diárias da maioria dos académicos no mundo.

Artigos epistemológicos e metodológicos confrontam-nos com desafios que vão para lá das decisões práticas e imediatas, obrigando-nos ao mesmo tempo a dar um passo atrás para observar as nossas opções com mais tempo, de maneira a sermos capazes de as avaliar criticamente. Este momento de reflexão permitirá aos investigadores compreender a relevância das suas soluções específicas, ampliando perspetivas analíticas e explorando aspetos relevantes da sua pesquisa, que, normalmente, ficam de fora do enquadramento dominante, orientado para decisões de curto prazo. Um olhar breve pelo conteúdo deste número demonstra que não podemos associar estes contributos metodológicos e epistemológicos apenas a tópicos de investigação menos recentes, visto que estas questões clássicas aplicam-se às inovações atualmente desenvolvidas nas sociedades modernas. Explorar a forma como a investigação é conduzida no contexto global do Big Data ou nos espaços omnipresentes das redes sociais online são apenas duas indicações da necessidade de construir as condições indispensáveis para manter o diálogo entre questões de curto e longo prazo acerca da forma como a nossa investigação é conduzida (Hutchinson, 2016).

A evolução tecnológica em curso no setor dos média e da comunicação também está a afetar os desenhos de investigação e as práticas neste campo. O nosso conjunto de ferramentas analíticas pode ainda incluir algumas das questões e abordagens teóricas clássicas, mas a transformação digital e a mudança computacional que tem sido vivida desde, pelo menos, a transição para o século XXI está obviamente a redefinir o nosso papel e hábitos em termos de investigação científica. Ensinar na área dos Estudos dos Média e das Metodologias de Investigação em Comunicação requer que nos mantenhamos a par destas inovações, mas de forma equilibrada, que não descure as 
questões centrais clássicas e ainda relevantes acerca do papel desempenhado pela comunicação em vários tipos de dinâmicas sociais. Como poderá ser alcançado, e, se possível, potenciado, este equilíbrio? Este é um dos principais desafios com que se depara a investigação em Comunicação, enquanto campo científico e/ou disciplina: avançar, com uma confiança intelectual crescente e resultados empíricos, por um caminho cheio de novos estímulos externos que desafiam as nossas principais questões de investigação, em termos de como e por que motivo tentamos resolvê-las. É, por isso, essencial manter uma abordagem crítica ao explorar ambientes digitais, por forma a assegurar o nosso papel como cientistas sociais e cidadãos dedicados. Se este número da Comunicação $e$ Sociedade nos ajudar a aproximar-nos de algumas das respostas a essas questões, então o esforço de criar um conjunto coerente de artigos interessantes terá valido a pena.

É possível perceber, a partir das reflexões que constituem este número da Comunicação e Sociedade, que o campo das Metodologias é, cada vez mais, encarado como um espaço de diálogo, e por vezes de tensão, entre os diversos paradigmas (ou abordagens) de investigação e teorias da comunicação. Quando consideramos o vasto campo que articulam, constituído pela Epistemologia e Filosofia da Ciência, pela Ética e pela vertente mais operativa dos métodos e das técnicas, temos um espaço amplo de debate acerca dos seus usos e potencialidades, mas também dos desafios que todas estas dimensões colocam a quem investiga em Comunicação.

O reconhecimento da importância das Metodologias de Investigação ocorre também no âmbito do ensino da Comunicação, através da integração de unidades curriculares desta natureza nos planos de estudos, uma realidade que, todavia, poderá variar consoante os contextos. No caso de Portugal e Espanha, por exemplo, é possível perceber-se uma redução do peso das Ciências Sociais e Humanas nos curricula dos cursos de Comunicação e Jornalismo, o que acarreta, necessariamente, um decréscimo da presença das Metodologias (Sanchéz-García \& Marinho, 2015).

Quando este reconhecimento ocorre, a existência de conteúdos dedicados à Metodologia e as próprias abordagens que estes conteúdos favorecem são testemunho, muitas vezes, da orientação epistemológica que preside à criação dos planos de estudo dos cursos e da forma como se enquadram no campo das Ciências Sociais e Humanas. Neste número da revista Comunicação e Sociedade, procurou-se abrir espaço a ambas as realidades - a do ensino e a da investigação - e à diversidade de posicionamentos epistemológicos que caracterizam o campo.

Um outro indicador do relevo atribuído às Metodologias passa pela sua representatividade entre as revistas científicas. Sem termos qualquer pretensão de exaustividade na sua identificação, é possível encontrar diversas publicações centradas nos "métodos quantitativos" e "métodos qualitativos", no tema da "ética em investigação" e em torno da "epistemologia", "filosofia da ciência" ou de métodos específicos, como é o caso da Action Research Journal, dedicada à "investigação-ação". O número reduz-se drasticamente, se nos centrarmos no campo da Comunicação. Aqui, destacamos a Communication, Methods and Measures ou, de âmbito mais restrito, a Journal of Information Communication and Ethics in Society. Se estendermos o raciocínio às associações 
científicas, mantém-se o panorama de uma atenção reduzida à Metodologia de Investigação: a IAMCR (International Association for Media and Communication Research) não tem qualquer secção dedicada ao tema; o que a ICA (International Communication Association) tem de mais próximo é uma divisão de "Philosophy, theory and critique" e um "interest group" de "Computational Methods"; na mesma linha, a ECREA (European Communication Research and Education Association) tem uma secção de "Philosophy of Communication" e destaca-se aqui a AEJMC (Association for the Education in Journalism and Mass Communication), com uma divisão de "Communication Theory and Methodology". No contexto lusófono e ibero-americano parece haver uma maior preocupação com estas questões: a ALAIC (Associação LatinoAmericana de Investigadores em Comunicação) tem um grupo de trabalho dedicado à "Teoria e Metodologia da Investigação em Comunicação"; a Assibercom (Associação Ibero-americana de Comunicação) dedica uma divisão temática à "Epistemologia, Teoria e Metodologia da Investigação"; e a Intercom (Sociedade Brasileira de Estudos Interdisciplinares da Comunicação) tem um grupo de pesquisa em "Teorias da Comunicação", com enfoque epistemológico/metodológico. No congresso de 2017, a Agacom (Asociácion Galega de Investigadores e Investigadoras en Comunicación) incluiu no programa uma mesa temática de "Teorías, métodos e técnicas de investigación da comunicación e da recepción" e, em 2016, no congresso da Lusocom (Federação Lusófona de Ciências da Comunicação) houve um grupo de trabalho sobre "Epistemologia da Comunicação" (o que já não se vê no programa do congresso de 2018). Já a Sopcom (Associação Portuguesa de Ciências da Comunicação) não tem qualquer grupo de trabalho dedicado à Epistemologia e Metodologia da Comunicação.

Anotamos ainda a preocupação que se tem registado em olhar para as Metodologias de Investigação de um outro ponto de vista: procurando aferir que abordagens, metodologias, métodos e técnicas são mais utilizados por quem publica na área das Ciências da Comunicação. Apenas a título de exemplo, podemos citar o trabalho de Trumbo (2004), que avaliou as edições de oito das mais relevantes revistas internacionais na área, entre 1990 e 2000 , mas existem outras publicações similares, algumas delas pioneiras neste esforço de meta-análise do campo (Brashers \& Jackson, 1999; Cooper, Potter \& Dupagne, 1994; Emmers-Sommer \& Allen, 1999; Kamhawi \& Weaver, 2003; Lowry, 1979; Perloff, 1976; Riffe \& Freitag, 1997; Schramm, 1957; Weaver, 1988; Weaver, 1993; Wimmer \& Haynes, 1978 citados por Trumbo, 2004).

É neste contexto, o de um caminho ainda a fazer para aumentar a representatividade das Metodologias de Investigação em Comunicação, que trazemos à luz esta edição da Comunicação e Sociedade, com o intuito de dar um pequeno contributo para este debate tão necessário, esperando que possa tornar-se mais frequente. Passamos, então, a apresentar esta edição.

O número abre com um texto ensaístico, em que Cicilia Peruzzo, servindo-se a sua experiência como investigadora na área, com especial interesse no campo das metodologias, traça um panorama crítico dos usos e desenvolvimento da Epistemologia e Metodologia na investigação em Ciências da Comunicação no Brasil. Segue-se um artigo 
em que Lumárya Souza de Sousa e Thaiane Oliveira fundamentam a possibilidade de a investigação-ação se poder constituir como o eixo agregador de uma epistemologia do Sul, a partir de uma cartografia sobre o uso deste método, também no contexto brasileiro. Abrimos, por isso, este volume com dois textos de nível macro, que discutem paradigmas de investigação e questões epistemológicas transversais da pesquisa em Comunicação.

Continuamos com quatro textos que abordam as Metodologias de Investigação, mas já centradas em campos específicos dos estudos em Comunicação, a um nível meso. Francisco Paulo Jamil Marques e Edna Miola analisam a produção científica na área da Comunicação e Política, no Brasil, para identificarem os seus progressos e obstáculos epistemológicos e metodológicos mais relevantes e avançam algumas propostas para ultrapassar esses entraves. Seguimos com dois artigos sobre Big Data e análise de rede, ambos com perspetivas críticas em relação às abordagens metodológicas que poderão/deverão ser adotadas nestas áreas. Ana Thereza Nogueira Soares fundamenta um posicionamento crítico acerca das implicações epistemológicas, metodológicas e teóricas das investigações com Big Data (em particular as que recorrem a dados dos média sociais) para o campo científico da comunicação, com especial enfoque na necessidade de valorizar o domínio teórico nesta área. Também Francisco Conrado Filho e Luís António Santos evidenciam a importância das perspetivas teóricas e conceptuais para a Análise de Redes Sociais (ARS) e, para isso, conduzem-nos pelas diversas abordagens que podem ser adotadas, passando pelas suas mais-valias e riscos. Este é, pois, um artigo que se reveste de uma natureza também pedagógica, já que poderá funcionar como uma leitura introdutória para quem esteja a iniciar um percurso na ARS. Fechamos este nível meso com um contributo de natureza ensaística de Pedro de Andrade sobre o Turismo Cultural Urbano, em que o autor faz um percurso pelas principais abordagens metodológicas tidas por referência no campo da Sociologia Artística para defender uma nova abordagem, assente nos conceitos de "hibridação" e "culturas móveis".

Os dois artigos que se seguem centram-se na discussão de procedimentos específicos, já discussões de nível micro no contexto de abordagem às Metodologias de Investigação que aqui adotamos. Ruth de Frutos e Diego Giannone preocupam-se em avaliar os desafios metodológicos colocados pelos dois indicadores mais importantes para medir a liberdade de imprensa no mundo - Freedom House e Repórteres Sem Fronteiras -, para mostrar em que medida esses instrumentos apresentam deficiências, nomeadamente a ausência de mecanismos de proteção dos jornalistas como variável. O contributo de Rita de Cássia Romeiro Paulino e Marina Lisboa Empinotti consiste na fundamentação teórico-metodológica do processo de construção de um modelo para avaliar a produção de conteúdos jornalísticos (notícias) para dispositivos móveis (smartphones), usando uma interface do tipo "cartão".

O autor Cláudio Márcio Magalhães traz-nos o único artigo deste número da Comunicação e Sociedade eminentemente centrado no ensino. Não se trata do ensino propriamente dito de Metodologias de Investigação, mas antes do desenvolvimento de uma estratégica pedagógica (com recurso a técnicas de investigação) para ensinar um conceito 
teórico (neste caso, a motivação), no âmbito de uma disciplina da área da Comunicação (mais especificamente da área da Publicidade e Propaganda).

A secção de artigos fecha com um contributo centrado na Ética, uma área primordial e transversal para as Metodologias de Investigação. Fernanda Pires de Sá discute as inquietações e desafios éticos que se colocam ao longo de um percurso de investigação (neste caso, sobre a covisualização de uma telenovela), para evidenciar que as decisões sobre questões éticas devem ser ponderadas não só no início de uma investigação, mas também ao longo de cada etapa do processo, num processo contínuo de ajustamento.

O volume termina com duas leituras, uma, da autoria de Eduardo Francisco Rodríguez Gómez, sobre um livro de Metodologias de Investigação em Comunicação, um manual de síntese e mapeamento do campo, que procura fazer a ponte entre os Métodos de Investigação e as Teorias da Comunicação; e a outra, da autoria de Madalena Oliveira, sobre um livro especificamente dedicado a tratar perspetivas (trata-se de uma obra coletiva) de análise da rádio. Se a primeira obra procura constituir-se como uma abordagem diferente numa área em que há já uma consistente produção (a dos manuais de Metodologia de Investigação), a segunda resulta num contributo para uma área em que a produção é escassa, quase inexistente.

Em 2007, no primeiro número da revista Communication, Methods and Measures, afirmava-se a "necessidade clara e defensável" (Roskos-Ewoldsen, Aakhus, Hayes, Heider \& Levine, 2007, p. 2) de uma publicação exclusivamente dedicada à Metodologia na área da Comunicação, uma ideia refirmada dez anos depois, na mesma revista, em jeito de balanço (Matthes, Niederdeppe \& Shen, 2016). Por partilharmos esta perspetiva - a de que é crucial fomentar o debate em torno das Metodologias de Investigação no campo das Ciências da Comunicação - entendemos relevante a edição deste número da Comunicação e Sociedade. Não porque a temática tenha estado ausente das páginas da revista - tal como os restantes periódicos científicos internacionais, publica artigos centrados nas questões metodológicas (Costa, 2000; Damásio, Henriques \& Mackart, 2012; F. Lopes, 2000; M. I. V. Lopes, 2009) -, mas porque consideramos que esta importância não deverá ser apenas episódica.

\section{REFERÊNCIAS BIBLIOGRÁFICAS}

Castells, M. (2000). The rise of the network society. The information age: economy, society and culture. Hoboken, Nova Jersy: John Wiley \& Sons

Costa, M. S. (2000). As ciências da comunicação: consolidação académica e desafios interdisciplinares. Comunicação e Sociedade, 2, 71-76. doi: 10.17231/comsoc.2(2000).1383

Craig, R. T. (1999). Communication theory as a field. Communication Theory, 9(2), 119-161. doi: 10.1111/j.14682885.1999.tboo355.x

Craig, R. T. (2008). Communication in the conversation of disciplines. Russian Journal of Communication, 1(1), 7-23. doi: 10.1080/19409419.2008.10756694 
Damásio, M. J., Henriques, S. \& Mackert, M. (2012). Saúde electrónica e literacia em saúde: uma revisão da metodologia de pesquisa. Comunicação e Sociedade (Vol. Especial), 171-183. doi: 10.17231/ comsoc.23(2012).1362

Hutchinson, J. (2016). An introduction to digital media research methods: how to research and the implications of new media data. Communication Research and Practice, 2(1), 1-6. doi: $10.1080 / 22041451.2016 .1155307$

Lopes, F. (2009). Estudos televisivos: perspectivas diacrónicas. Comunicação e Sociedade, 15, 7-27. doi: $10.17231 /$ comsoc.15(2009).1042

Lopes, M. I. V. (2000). Metodologia para o estudo de recepção de telenovelas no Brasil. Comunicação $e$ Sociedade, 2, 93-112. doi: 10.17231/comsoc.2(2000).1386

Matthes, J., Niederdeppe, J. \& Shen, F. C. (2016). Reflections on the need for a journal devoted to communication research methodologies: ten years later. Communication Methods and Measures, 10(1), 1-3. doi: $10.1080 / 19312458.2016 .1136514$

Norderstreng, K. (2004). Ferment in the field: notes on the evolution of communication studies and their disciplinary nature. Javnost - The Public, 10(3), 5-18. doi: 10.1080/13183222.2004.11008856

Roskos-Ewoldsen, D., Aakhus, M., Hayes, A. F., Heider, D. \& Levine, T. (2007). It's about time: the need for a journal devoted to communication research methodologies. Communication Methods and Measures, 1(1), 1-5. doi: 10.1080/19312450709336657

Sanchéz- García, P. \& Marinho, S. (2015). La enseñanza universitaria encaminada a la especialización y al recorte de Humanidades: estudio de caso de la titulación de Periodismo en España y Portugal. RIESED - Revista Internacional de Estudios sobre Sistemas Educativos, 4, 17-36. Retirado de http://www.riesed.org/ index.php/RIESED/article/view/69

Trumbo, C. (2004). Research methods in mass communication research: a census of eight journals 19902000. Journalism and Mass Communication Quarterly, 81(2), 417- 436. doi: 10.1177/107769900408100212

\section{NOTAS BIOGRÁFICAS}

Sandra Marinho é doutorada em Ciências da Comunicação, na área de Estudos de Jornalismo, pela Universidade do Minho. É Professora Auxiliar nesta Universidade, onde ensina nas áreas de Metodologias de Investigação e Jornalismo.

Desenvolve investigação no Centro de Estudos de Comunicação e Sociedade (CECS) sobre a qualidade do jornalismo e do ensino do jornalismo e sobre jornalismo de saúde. Tem diversas publicações nestas áreas, em periódicos nacionais e internacionais.

E-mail:marinho@ics.uminho.pt

Morada: Centro de Estudos de Comunicação e Sociedade

Campus de Gualtar

4710-057 Braga, Portugal

Miguel Vicente Mariño é Professor e Diretor do Departamento de Sociologia e Trabalho Social da Universidade de Valladolid, na Faculdade de Ciências Sociais, Jurídicas e de Comunicação. Doutor em Comunicação Audiovisual, Bacharel em Sociologia e Bacharel em Jornalismo, é membro do Comité Executivo da ECREA (European 
Communication Research and Education Association), subdiretor da seção de Audiências da IAMCR (International Association for Media and Communication Research) e do Grupo de Trabalho sobre História da Pesquisa em Comunicação da AE-IC (Associação Espanhola de Pesquisa em Comunicação). As suas linhas de investigação são o estudo dos média e das audiências, a comunicação ambiental, os processos de formação da opinião pública nas sociedades atuais e os métodos de pesquisa aplicados ao estudo da comunicação.

E-mail: miguelvm@soc.uva.es

Morada: Universidad de Valladolid - Campus de Segovia

Plaza de la Universidad, 1. 40005 Segovia 